\title{
Cranial Nerve Abnormalities in Oculo-Auriculo-Vertebral Spectrum
}

\author{
R. Manara, D. Brotto, S. Ghiselli, R. Mardari, I. Toldo, G. Schifano, E. Cantone, R. Bovo, and A. Martini
}

\begin{abstract}
BACKGROUND AND PURPOSE: Cranial nerve abnormalities might be observed in hemifacial microsomia and microtia (oculo-auriculovertebral spectrum), but the rate, features, and relationship with functional impairment or phenotype severity have not yet been defined. This study aimed at investigating absence/asymmetry, abnormal origin, morphology and course of cranial nerves, and presence/asymmetry of the foramen ovale and inferior alveolar nerve canal in a cohort of oculo-auriculo-vertebral spectrum patients.
\end{abstract}

MATERIALS AND METHODS: Twenty-nine patients with oculo-auriculo-vertebral spectrum (mean age, 7 years; age range, 0.2-31 years; 12 females) underwent brain MR imaging, CT, and neurologic evaluation; 19 patients had a more severe phenotype (Goldenhar syndrome).

RESULTS: Cranial nerve abnormalities were detected only in patients with Goldenhar syndrome (17/19, bilaterally in 8$)$ and were involved the second (4/19), third (1/18), fifth (11/19), sixth (8/16), seventh (11/18), and eighth (8/18) cranial nerves. Multiple cranial nerve abnormalities were common (11/17). Eleven patients showed bone foramina abnormalities. Trigeminal and facial nerve dysfunctions were common (44\% and $58 \%$, respectively), especially in patients with Goldenhar syndrome. Trigeminal abnormalities showed a good correlation with ipsilateral dysfunction $(P=.018)$, which further increased when bone foramina abnormalities were included. The facial nerve showed a trend toward correlation with ipsilateral dysfunction $(P=.081)$. Diplopia was found only in patients with Goldenhar syndrome and was associated with third and sixth cranial nerve abnormalities $(P=.006)$.

CONCLUSIONS: Among patients with oculo-auriculo-vertebral spectrum, cranial nerve morphologic abnormalities are common, correlate with phenotype severity, and often entail a functional impairment. The spectrum of cranial nerve abnormalities appears wider than simple hypo-/aplasia and includes an anomalous cisternal course and partial/complete fusion of diverse cranial nerves.

ABBREVIATIONS: $\mathrm{CN}=$ cranial nerve; OAVS = oculo-auriculo-vertebral spectrum

O culo-auriculo-vertebral spectrum (OAVS) (Online Mendelian Inheritance in Man, 164210 $)^{1}$ is a rare heterogeneous congenital condition (incidence, 1:3500-5600 live births; male/ female ratio, $3: 2),{ }^{2-4}$ in which the head structures originating from the first and second pharyngeal arches are incompletely developed on 1 ( $85 \%$ of cases) or both sides. ${ }^{3,5}$ The disease mostly

\footnotetext{
Received October 7, 2014; accepted after revision November 29.

From Neuroradiology (R.Manara), University of Salerno, Salerno Italy; Departments of Neuroscience (D.B., S.G., G.S., R.B., A.M.) and Pediatrics (I.T.), University of Padova, Padova, Italy; Neuroradiology (R.Mardari), University Hospital of Padova, Padova, Italy; and Ear Nose and Throat Section (E.C.), Department of Neurosciences, "Federico II" University, Napoli, Italy.

Renzo Manara and Davide Brotto contributed equally to the study and should be considered co-first authors.

Please address correspondence to Renzo Manara, MD, Neuroradiology, University of Salerno, Via S Allende 1, 89081, Salerno, Italy; e-mail: rmanara@unisa.it

三 Indicates article with supplemental on-line tables.

Indicates article with supplemental on-line photo.

http://dx.doi.org/10.3174/ajnr.A4273
}

results in ear (microtia) and jaw (hemifacial microsomia) abnormalities (On-line Fig 1). Nonetheless, the abnormality spectrum might be fairly wide, from mild external and medium ear involvement or isolated facial asymmetry to anotia with complex facial deformity. The most severe cases also present with eye or spine involvement and are known as Goldenhar syndrome from the French ophthalmologist who first described the syndrome in 1952. ${ }^{6}$ Familial history suggestive of both autosomal recessive and dominant inheritance has been reported, and genes on chromosomes 5, 12, 14, and 22 have been implicated. ${ }^{7-10}$ However, most cases of OAVS are sporadic and without a known etiology. Abnormal embryonic vascular supply, ${ }^{11}$ hematomas, and drug use during the early phases of gestation have been reported to cause the disruption of mesodermal migration, leading to defective formation of bone and soft-tissue structures. ${ }^{12}$

Most interesting, a few case reports and small series studies have shown a concomitant impairment of cranial nerves (CNs), ${ }^{13-26}$ highlighting the possible involvement of neural struc- 
tures in OAVS and addressing its potentially relevant clinical impact. To date, the underlying anatomic and structural $\mathrm{CN}$ abnormalities have been poorly investigated because the available data rely on anecdotal postmortem examination ${ }^{17}$ or neuroimaging findings. ${ }^{19}$ Additionally, the overall frequency of $\mathrm{CN}$ abnormalities, their association with $\mathrm{CN}$ dysfunction, and the relationship with the OAVS phenotype severity have not yet been defined, to our knowledge.

In the past few years, MR imaging has become a powerful tool for investigating in vivo the cisternal segment of the CNs. With routinely available 1.5T MR imaging scanners and dedicated high-resolution sequences, it is possible to verify the presence and characterize the morphology, diameter, and cisternal course of most CNs. CN MR imaging evaluation has, therefore, become helpful for diagnosing several conditions such as Kallmann syndrome, optic neuritis, septo-optic dysplasia, neurovascular conflicts, and so forth. Moreover, the evaluation of the intrameatal branches of CN VIII is included in the diagnostic work-up of implant planning in patients with congenital profound hearing loss. Besides, because skull base foramina and bone canal development is induced by the presence of the corresponding $\mathrm{CN}$ branch, ${ }^{27} \mathrm{CN}$ abnormalities might be also indirectly inferred by bone CT. Absence or hypoplasia/stenosis of the facial canal, internal acoustic canal, foramina ovalia and rotundum, hypoglossal canal, or inferior alveolar canal might indicate hypoplasia or aplasia of the relative nerves and branches.

Therefore, MR imaging and CT might help to detect or raise the suspicion of morphologic $\mathrm{CN}$ abnormalities providing relevant information, especially when the $\mathrm{CN}$ impairment is difficult to evaluate due to early age, concomitant facial bone and soft-tissue asymmetry, or poor compliance of patients with OAVS.

The present study aims at investigating, in patients with OAVS, the rate of CN abnormalities, the type (eg, agenesia, hypoplasia, abnormal origin, or cisternal course), the association with functional impairment, and the side of hemifacial microsomia as well as the relationship with the phenotype severity.

\section{MATERIALS AND METHODS Subjects}

Twenty-nine patients (mean age, 7 years; age range, $0.2-31$ years; 12 females) with hemifacial microsomia and microtia were considered (On-line Table 1). Nineteen patients (mean age, 7.7 years; age range, $0.2-31$ years; 9 females) also presented with ocular or cervical vertebral abnormalities and fulfilled the diagnostic criteria for Goldenhar syndrome (abnormalities involving the face, ear, eye, and/or cervical vertebrae).

The phenotypic spectrum of patients with OAVS is presented in On-line Table 2.

\section{Neuroimaging}

All except 1 patient underwent 1.5T MR imaging; 3 patients underwent MR imaging in other centers, but the image quality was considered appropriate for the study purposes. Our hospital MR imaging study protocol (Achieva 1.5T; Philips Healthcare, Best, the Netherland) included the following: 1) high-resolution T2weighted imaging for the visualization of $\mathrm{CNs}$ (driven equilib- rium sequence; TR, $1500 \mathrm{~ms}$; TE, $250 \mathrm{~ms}$; FOV, $130 \mathrm{~mm}$; voxelsize, $0.6 \times 0.6 \times 0.6 \mathrm{~mm}$ ) covering the whole brain stem; 2) high-resolution volumetric T1-weighted imaging (TR, $20 \mathrm{~ms}$; TE, $5.8 \mathrm{~ms}$; voxel-size, $0.66 \times 0.66 \times 1 \mathrm{~mm}$ ) covering the whole brain. From both sequences, multiplanar reconstructions were generated along the 3 orthogonal planes. Contrast medium was never administered. The entire MR imaging examination lasted approximately 20-25 minutes. Patients younger than 5 years of age required sedation, except for some infants who underwent the examinations without sedation.

At MR imaging, the neuroradiologists assessed the presence/ absence of the CNs, their side-to-side diameter asymmetry on images orthogonal to their cisternal course, any anomaly regarding the origin from the brain stem, the morphology, and the cisternal course.

In this study, the evaluation was mostly based on T1-weighted images for CNs I-II and on T2-weighted images for CNs III-XI. The ninth and twelfth $\mathrm{CN}$ cisternal course is inconstantly recognizable at $1.5 \mathrm{~T}$ imaging, especially because these $\mathrm{CNs}$ are thin and there are several small vessels running in the same cisternal space; therefore, these nerves were not considered in the study. Regarding the inferior $\mathrm{CNs}$, the ninth-through-eleventh $\mathrm{CN}$ origin together with several roots from the retro-olivary sulcus, the number of roots might vary from subject to subject, thus usually making it impossible to recognize which root belongs to a specific nerve (except for the most cranial root that certainly belongs to CN IX).

All patients with OAVS had petrous bone or head CT. The study protocol varied greatly because several examinations were performed in other centers or in our center with different scanners; section thickness was $<1 \mathrm{~mm}(0.6 \mathrm{~mm}$ in 26 cases $)$ with subsequent $3 \mathrm{D}$ multiplanar reconstructions. Examinations were not repeated in patients with CT performed in different centers unless required for clinical or surgery management, even though in some cases not all bone structures were evaluable. In particular, CT allowed the assessment of the foramen ovale and the inferior alveolar bone canal in 25/29 and 19/29 patients, respectively.

CT evaluation focused on the internal, middle, or external ear abnormalities and the presence and symmetry of the foramen ovale and the inferior alveolar bone canal. Neuroradiologists assessed the presence/absence and the side-to-side asymmetry of the above-mentioned bone foramina.

Clinical information about the third-through-seventh $\mathrm{CN}$ function was obtained by the pediatrician or the family doctor of $20 / 29$ patients. In addition, the presence of anisocoria, diplopia, facial hypoesthesia (including corneal reflex), and masticatory muscle and upper and lower facial muscle weakness was assessed by neurologic evaluation. Visual and hearing abnormalities were obtained for all patients from clinical records.

All MRI and CT was evaluated in consensus by 2 experienced neuroradiologists (R.Manera, R.Mardari), blinded to the patients' clinical findings.

The study protocol was approved by our ethics committee, and all patients or parents of children gave written informed consent. 


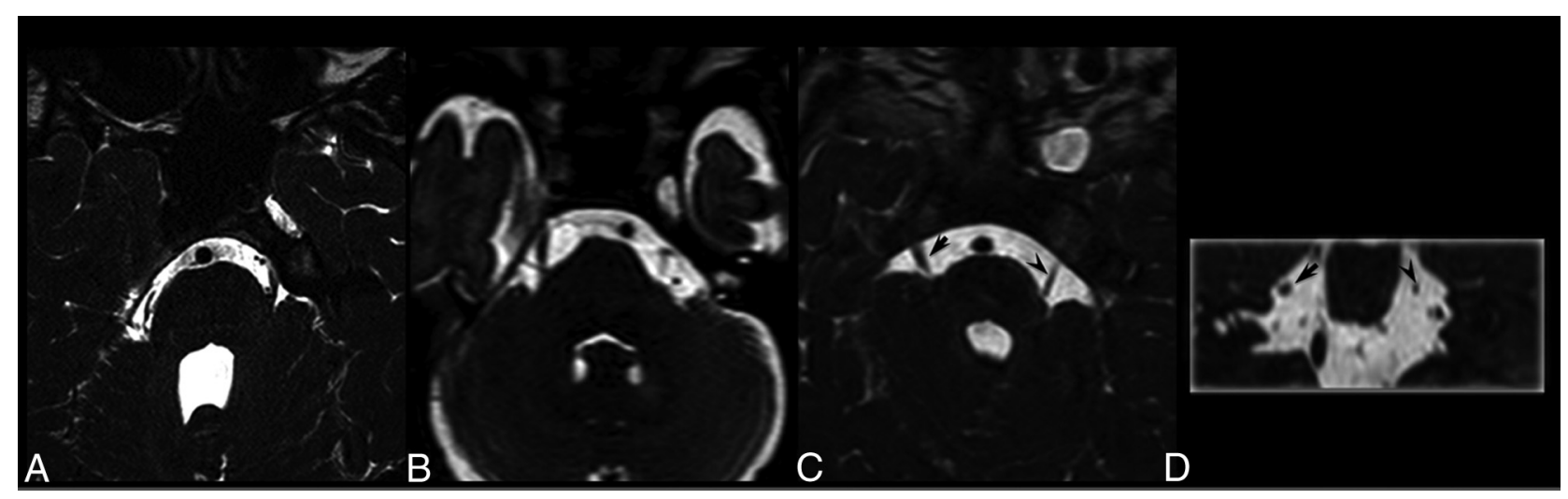

FIG 1. A-C, Axial T2-weighted images of 3 different patients with Goldenhar syndrome. $A$, The absence of the right trigeminal nerve. $B$, The absence of the left trigeminal nerve. $C$, The relative hypoplasia of the left trigeminal nerve (arrowhead). $D$, Coronal multiplanar reconstruction image, obtained from the same patient as in C, shows the different diameters of the trigeminal nerves (arrow and arrowhead).

\section{Statistical Analysis}

Study data were analyzed by the Fisher exact test; statistical significance was set at $P<.05$.

\section{RESULTS}

Findings on CNs and bone foramina are shown in On-line Table 3. In particular, 17/29 (59\%) patients presented with intracranial $\mathrm{CN}$ abnormalities, and 11 patients showed abnormalities of the bone foramina of the trigeminal branches (foramen ovale, inferior alveolar nerve bone canal). Two patients presented with isolated bone foramen abnormalities without $\mathrm{CN}$ abnormalities at MR imaging, and 2 patients had abnormalities of the foramina without ipsilateral trigeminal nerve abnormalities. Among the 19 patients with intracranial or distal $\mathrm{CN}$ involvement (27 sides, 8 patients had bilateral $\mathrm{CN}$ involvement), the abnormalities were ipsilateral to facial microsomia in $23 / 27$ cases; in $3 / 4$ of the remaining patients, the $\mathrm{CN}$ abnormalities were bilateral, despite unilateral facial microsomia.

The trigeminal nerve (Fig 1) was the most frequently involved (11 patients, bilaterally in 5), followed by CN VII (11 subjects, bilaterally in 3) and CNs VI and VIII ( 8 patients, bilaterally in 3 and 2, respectively). MR imaging disclosed CN III hypoplasia in 1 patient. Five patients had micro- or anophthalmia; 4 had ipsilateral optic nerve hypoplasia; and in 1, the optic nerves were not evaluable. Among patients with globe abnormalities, only 1 had CN III and VI ipsilateral abnormalities, while in 1 patient, these CNs were not evaluable. No morphologic abnormalities of the lower $\mathrm{CNs}$ were found. Multiple $\mathrm{CN}$ abnormalities (up to 7 $\mathrm{CNs})$ were found in most $(11 / 17)$ patients. Among the 9 patients with foramen ovale hypo-/aplasia, 2 had normal trigeminal nerves at MR imaging; among the 8 patients with alveolar nerve bone canal hypo-/aplasia, 3 had a normal trigeminal nerve at MR imaging (On-line Fig 2). If we considered the 16 abnormal trigeminal nerves, 8 had ipsilateral bone foramina abnormalities (in all, the foramen ovale was abnormal; 5 had inferior alveolar bone canal abnormalities, while in 3 , the inferior alveolar bone was not evaluable); 6 did not have ipsilateral bone foramina abnormalities; and in 2, CT did not allow foramina evaluation. Among the 9 patients with foramina ovalia abnormalities ( 1 patient had no foramina ovalia bilaterally), the ipsilateral inferior alveolar bone canal was abnormal in 6 cases, normal in 1 , and not evaluable in 3 .

In our cohort, $\mathrm{CN}$ abnormalities at $\mathrm{MR}$ imaging were found only in patients with Goldenhar syndrome (17/19), while no patient with a mild phenotype $(0 / 10)$ presented with abnormal CNs $(P=.000)$. The association with the Goldenhar phenotype persisted when considering the trigeminal nerve abnormalities, either without $(11 / 18$ versus $0 / 10, P=.002)$ or with foramen anomalies, consistent with involvement of trigeminal branches (13/18 versus $1 / 10, P=.004)$. Similarly, the sixth, seventh, and eighth $\mathrm{CN}$ abnormalities were significantly associated with the Goldenhar phenotype compared with patients with the mild phenotype $(8 / 16$ versus $0 / 10, P=.009 ; 11 / 17$ versus $0 / 10, P=.001$; and $8 / 18$ versus $0 / 10, P=.025$, respectively).

Considering that some $\mathrm{CN}$ evaluations were not reliable because of patient age, compliance, or concomitant severe facial asymmetry among the 20 patients ( 11 with Goldenhar phenotype) in whom the functional evaluation was performed, facial nerve and trigeminal dysfunctions were found in 11/19 and 7/16 patients, respectively. Facial nerve and trigeminal dysfunctions were significantly more frequent in patients with Goldenhar syndrome ( $9 / 10$ with Goldenhar phenotype versus $2 / 9$ with mild phenotype, $P=.0054$; and $6 / 8$ with Goldenhar phenotype versus $1 / 8$ with mild phenotype, $P=.041$, respectively). Oculomotor dysfunction was found in 4 patients ( 2 patients were not considered due to anophthalmia, which hampered the evaluation of oculomotor function), all with Goldenhar syndrome, showing a trend of association with the phenotype (4/9 with Goldenhar phenotype versus $0 / 9$ with mild phenotype, $P=.082$ ).

MR imaging of 1 patient with trigeminal dysfunction ipsilateral to hemifacial microsomia was not reliably evaluable for $\mathrm{CN} \mathrm{V}$. Among the remaining 15 patients with trigeminal function evaluation (30 trigeminal nerves), 5 had trigeminal nerve morphologic abnormalities ( 2 bilaterally, ie, 7 abnormal trigeminal nerves). Trigeminal dysfunction was associated with ipsilateral trigeminal nerve morphologic abnormalities (4/7 versus $2 / 23$, $P=$.016). Of the 2 patients with no trigeminal nerve morphologic abnormalities but with trigeminal nerve dysfunction, the inferior alveolar nerve bone canal was absent in 1 patient and not evaluable in the other.

AJNR Am J Neuroradiol 36:1375-80 Jul 2015 www.ajnr.org 


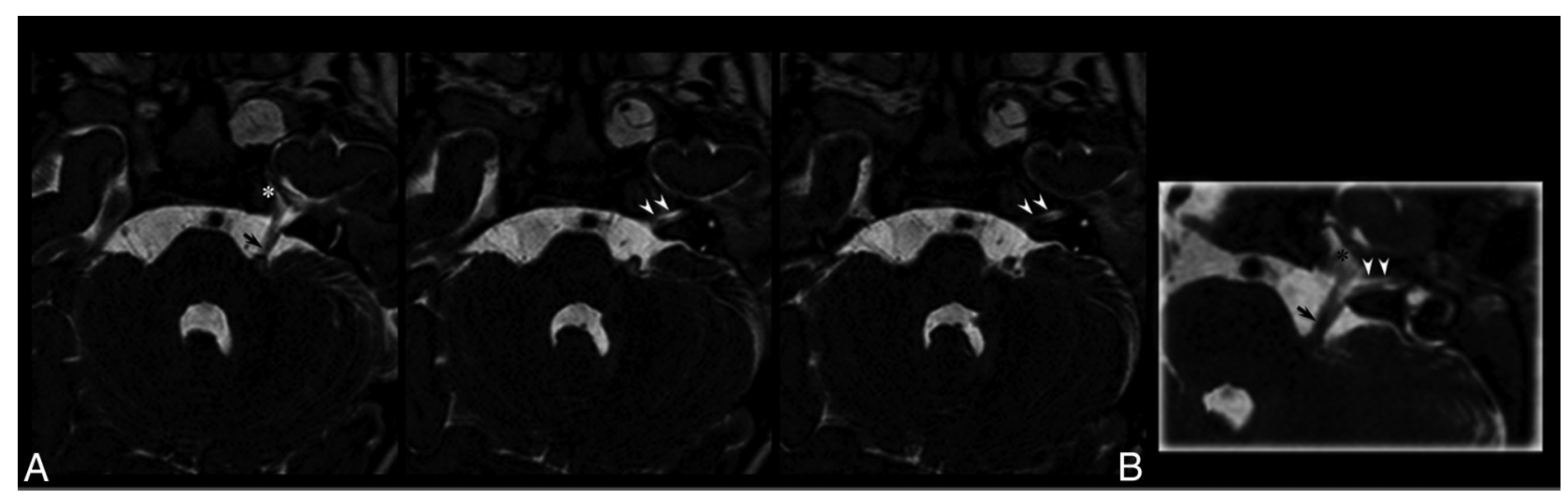

FIG 2. Brain MR imaging. A, Contiguous T2-weighted axial images show the common origin of the left fifth and seventh/eighth cranial nerves from the anterior profile of the middle cerebellar peduncle (arrow). The common trunk runs in the cisternal space and subdivides into 2 nerves entering the Meckel cave (asterisk) and, slightly caudally, the internal acoustic meatus (arrowheads). B, Oblique multiplanar reconstruction shows the common nerve origin and the subsequent subdivision.

Among the 18 patients (36 sides) with functional oculomotor evaluation, 4 had morphologic CN VI abnormalities (bilaterally in 2, ie, 6 abnormal abducens nerves). Oculomotor dysfunction (bilateral in 1 patient, unilateral in 3) was associated with ipsilateral CN VI morphologic abnormalities (3/6 versus $2 / 30, P=$ $.024)$.

Finally, 1 patient with Goldenhar syndrome underwent multiple facial surgeries, and facial function was, therefore, not reliably evaluable. Among the remaining 19 patients (38 sides), 6 had morphologic CN VII abnormalities (bilateral in 2). Facial nerve dysfunction (bilateral in 2 patients, unilateral in 9) showed a trend toward association with ipsilateral nerve abnormalities (5/8 versus $8 / 30, P=.081)$.

\section{DISCUSSION}

This neuroradiologic study showed the strikingly common involvement of CNs among patients with oculo-auriculo-vertebral spectrum, especially among those presenting with the more severe phenotype (Goldenhar syndrome). In our series, at MR imaging, $17 / 29$ patients (59\%) displayed 57 abnormal CNs ranging from hypoplasia/aplasia to protean morphologic abnormalities; moreover, at CT, 4 patients showed isolated hypoplasia of the foramen ovale and/or of the inferior alveolar nerve bone canal, consistent with trigeminal branch hypo-/aplasia, thus disclosing also a possible distal $\mathrm{CN}$ involvement.

$\mathrm{CN}$ involvement in OAVS has been previously clinically and radiologically described in case reports or small series studies. ${ }^{13-26}$ In 1975, a pathologic study on a child affected with Goldenhar syndrome $^{17}$ showed the absence of the cisternal portion of the right trigeminal nerve along with the absence of its intra-axial nuclei, providing direct evidence of $\mathrm{CN}$ and central nervous system involvement in patients with OAVS. Moreover, because skull base foramina development is influenced by the existence of the corresponding $\mathrm{CN}$ branches, ${ }^{27} \mathrm{CN}$ abnormalities might be also be indirectly inferred by skull evaluation. The presence of hypoplastic and deformed foramina ovalia and rotundum in a paleopathologic case affected with Goldenhar syndrome ${ }^{28}$ seems to confirm the possible concomitant distal involvement of the trigeminal branches (S. Panzer, MD, personal e-mail communication, January 1, 2013). Similar evaluation can be performed by CT, which allows the precise visualization of the bone foramina. In the present study, 11/26 (42\%) patients with OAVS with adequate bone CT showed foramina abnormalities, suggesting that the distal involvement of the trigeminal branches is not uncommon.

In the past few years, MR imaging has provided high-resolution images suitable for the evaluation in vivo of the CNs, especially at the cisternal portion, thus contributing, in some cases, to the explanation of their absent or impaired function. ${ }^{29,30} \mathrm{Al}$ though CNs IV and XII might be inconstantly recognizable at 1.5T and CNs IX-XI appear as an indistinguishable group of roots originating from the retro-olivary sulcus, MR imaging reliably assesses the absence or the relative hypoplasia of the remaining CNs. Thus, MR imaging has become a cornerstone in the diagnostic work-up of children with profound congenital deafness, helping in cochlear or brain stem implantation decision-making. ${ }^{31}$ In fact, the absence of the cisternal segment of the eighth $\mathrm{CN}$ or the intrameatal absence of the cochlear nerve is considered, to date, a major contraindication to cochlear implantation.

At MR imaging, besides CN aplasia (absence of the cisternal segment) or hypoplasia (significant side-to-side asymmetry of the nerve diameter), in our study population, we recognized protean morphologic changes never reported in previous publications. One patient showed a nodular thickening of the fifth CN close to its origin (On-line Fig 3). Because the distal part of the nerve was recognizable and the mass diagnosis could not be supported by histology, this patient with Goldenhar syndrome was, as a precaution, not included among those with $\mathrm{CN}$ abnormalities. In some patients, a single nerve was rooted from the brain stem and was divided subsequently into different CNs. In 2 patients, for example, we did not detect the cisternal portion of the facial nerve concomitant to an enlarged ipsilateral trigeminal nerve and partially preserved facial nerve function. While direct pathologic evidence was not available, these cases likely represent the abnormal fusion of the cisternal segments of the facial and trigeminal nerves. Indeed, in one of these cases, an abnormal facial bone canal/internal acoustic meatus seemed to be recognizable close to the site where the abnormal CN V reached the upper surface of the petrous bone apex (Fig 2). Similarly, in 2 patients, there was only $1 \mathrm{CN}$ in the internal acoustic meatus, a condition that is consid- 
ered indicative of a preserved facial nerve with CN VIII aplasia ${ }^{31}$; nonetheless, both patients had partially preserved auditory function. The presence of nerve fibers within a different nerve or nerve branch has been suggested in some cases in which the absence of the cochlear nerve did not correspond to a complete loss of hearing function. ${ }^{32}$ Therefore, these cases seem to increase the body of evidence against the "dogmatic" indication toward auditory brain stem implantation whenever cranial VIII or the cochlear nerves are missing at MR imaging investigation, ${ }^{33}$ unless concomitant and coherent functional data are obtained.

In the current study, anomalies of CN II were not further considered because hypoplasia of the optic nerve was the rule when the patients presented with microphthalmia or anophthalmia. In contrast, abnormalities of the nerves that control eye movements did not correlate with abnormal eye development. Globe abnormalities were not significantly more frequent among patients with CN III or VI abnormalities (2/8 versus $3 / 18)$; this finding confirms that eye and oculomotor apparatus follow different development pathways. From a functional point of view, the association between Goldenhar syndrome and extrinsic ocular-movement impairment has been frequently noted. Duane syndrome type I has been reported in patients with Goldenhar syndrome. ${ }^{15,20-26}$ Among our patients with functional evaluation, 4/18 had oculomotor impairment (all Goldenhar phenotypes), while $8 / 28$ with MR imaging evaluation presented with CN III or VI abnormalities. Considering that this study did not evaluate $\mathrm{CN}$ IV morphologic abnormalities and that hypoplasia of CN VI is difficult to ascertain because the nerve is very thin, our data confirm the frequent involvement of the oculomotor nerves in OAVS and explain the frequent oculomotor impairment in these patients. On the other hand, the recurrent observation of preserved oculomotor function despite CN VI aplasia seems to support the hypothesis that nerve fibers might run along alternative routes to innervate the extrinsic ocular muscles.

From a pathogenic point of view, involvement of the CNs in OAVS is likely due to the common embryologic origin of facial bones, soft tissues, and peripheral neural structures from the first and second pharyngeal arches. These structures are contiguous to the corresponding neural crests and central nervous system structures. Whatever the nature of the lesion leading to OAVS (ischemic, hemorrhagic, infectious, and so forth), $\mathrm{CN}$ development might be concomitantly affected: In fact, most $\mathrm{CN}$ abnormalities were ipsilateral to facial abnormalities. On the other hand, in our cohort, a few patients showed bilateral $\mathrm{CN}$ involvement despite unilateral facial involvement. This observation suggests that neural structures in OAVS might be even more vulnerable than contiguous bone and soft tissues or that the clinical evaluation of microsomia (which is based on side-to-side comparison) might be less sensitive than the neuroradiologic or functional evaluation of the CNs.

Notably, the present study showed a strong association between Goldenhar phenotype (severe cases among the OAVS) and $\mathrm{CN}$ involvement. This might appear obvious because among the OAVS, Goldenhar syndrome criteria imply a more extensive involvement (facial and auricular involvement plus ocular or vertebral involvement). Nonetheless, we did not find within the Goldenhar subgroup an association between the number of fulfilled criteria and the number of involved CNs. Patients with Goldenhar syndrome with both ocular and vertebral involvement did not show an increased number of abnormal CNs compared with patients with Goldenhar syndrome with isolated ocular or vertebral involvement. A likely explanation is that the inclusion criteria for Goldenhar syndrome do not take into account the severity of ocular or vertebral abnormalities. Besides anophthalmia and microphthalmia, coloboma, epibulbar dermoid, or even epicanthus are considered among the inclusion criteria for ocular involvement in Goldenhar syndrome, though their clinical impact and severity are clearly different.

The clinical impact of $\mathrm{CN}$ abnormalities in patients with OAVS has been underlined in previous studies ${ }^{13-26}$; the present study on a relatively large sample shows that MR imaging and CT might provide the explanation of nerve impairment. Moreover, because neonates and infants might be difficult to evaluate and $\mathrm{CN}$ dysfunction might imply serious complications (eg, corneal lesions or abnormal masticatory dynamic), neuroimaging-focused evaluation might help when $\mathrm{CN}$ impairment is suspected in the early phases of the life of a patient with OAVS, thus helping to prevent irreversible damage. For this purpose, MR imaging is certainly the preferred tool, but in some cases, the evaluation of the foramen ovale and the inferior alveolar nerve bone canal might disclose $\mathrm{CN}$ abnormalities, even when the cisternal portion of the trigeminal nerve appears normal.

\section{CONCLUSIONS}

This study showed the following: 1) the frequent involvement of CNs in patients with OAVS spectrum, 2) the strong association between $\mathrm{CN}$ abnormalities and the Goldenhar phenotype, and 3) the association between $\mathrm{CN}$ abnormalities and functional impairment. Moreover, our findings widened the spectrum of $\mathrm{CN}$ abnormalities that is not limited to hypo-/aplasia but includes the anomalous course of the cisternal segment and the partial or complete fusion of $\geq 2 \mathrm{CNs}$. Further studies are warranted to investigate the possible role of genetics in the OAVS and the coexistence of brain, internal carotid artery, and inner ear involvement, which will probably help in understanding the likely pathogenesis of this condition.

\section{ACKNOWLEDGMENTS}

We are very grateful to Dr S. Panzer for her kindness and collaboration in providing interesting additional information about skull abnormalities of the paleopathologic case published in 2008.

Disclosures: Renzo Manara—UNRELATED: Payment for Lectures (including service on Speakers Bureaus): BioMarin, Serono, Comments: approximately $€ 2000$ (US \$2259.05).

\section{REFERENCES}

1. Online Mendelian Inheritance in Man. Goldenhar syndrome. McKusick-Nathans Institute for Genetic Medicine, John Hopkins University (Baltimore) and National Center for Biotechnology Information, National Library of Medicine (Baltimore). http://www. omim.org/entry/164210? search= goldenhar\%20syndrome\&highlight= goldenhar\%20syndrome\%20syndromic. Accessed September 12, 2009

2. Poswillo D. Otomandibular deformity: pathogenesis as a guide to reconstruction. J Maxillofac Surg 1974;2:64-72

AJNR Am J Neuroradiol 36:1375-80 Jul 2015 www.ajnr.org 
3. Grabb WC. The first and second branchial arch syndrome. Plast Reconstr Surg 1965;36:485-508

4. Rollnick BR, Kaye CI, Nagatoshi K, et al. Oculoauriculovertebral dysplasia and variants: phenotypic characteristics of 294 patients. Am J Med Genet 1987;26:361-75

5. Mastroiacovo P, Corchia C, Botto LD, et al. Epidemiology and genetics of microtia-anotia: a registry based study on over one million births. J Med Genet 1995;32:453-57

6. Goldenhar M. Associations malformatives de l'oeil et de l'oreille en particulaire, le syndrome dermoide epibulbaire-appendices auriculares-fistula auris congenital et ses relations avec le dysostose mandibulo-faciale. J Genet Hum 1952;1:243-82

7. Ala-Mello S, Siggberg L, Knuutila S, et al. Further evidence for a relationship between the $5 \mathrm{p} 15$ chromosome region and the oculoauriculovertebral anomaly. Am J Med Genet A 2008;146A: 2490-94

8. Rooryck C, Stef M, Burgelin I, et al. Mb terminal deletion in 12p13.33 associated with oculoauriculovertebral spectrum and evaluation of WNT5B as a candidate gene. Eur J Med Genet 2009; 52:446-49

9. Ballesta-Martínez MJ, López-González V, Dulcet LA, et al. Autosomal dominant oculoauriculovertebral spectrum and 14q23.1 microduplication. Am J Med Genet A 2013;161A:2030-35

10. Torti EE, Braddock SR, Bernreuter K, et al. Oculo-auriculo-vertebral spectrum, cat eye, and distal 22q11 microdeletion syndromes: a unique double rearrangement. Am J Med Genet A 2013;161A: 1992-98

11. Ottaviano G, Calzolari F, Martini A. Goldenhar syndrome in association with agenesia of the internal carotid artery. Int J Pediatr Otorhinolaryngol 2007;71:509-12

12. Hartsfield JK. Review of the etiologic heterogeneity of the oculoauriculo-vertebral spectrum (hemifacial microsomia). Orthod Craniofac Res 2007;10:121-28

13. Sugar HS. The oculoauriculovertebral dysplasia syndrome of Goldenhar. Am J Ophthalmol 1966;62:678-82

14. van Bijsterveld OP. Unilateral corneal anesthesia in oculoauriculovertebral dysplasia. Arch Ophthalmol 1969;82:189-90

15. Bowen DI, Collum LM, Rees DO. Clinical aspects of oculo-auriculovertebral dysplasia. $\mathrm{Br} J$ Ophthalmol 1971;55:145-54

16. Budden SS, Robinson GC. Oculoauricular vertebral dysplasia: its association with sensorineural deafness and other abnormalities. Am J Dis Child 1973;125:431-33

17. Aleksic S, Budzilovich G, Reuben R, et al. Congenital trigeminal neuropathy in oculoauriculovertebral dysplasia-hemifacial microsomia (Goldenhar-Gorlin syndrome). J Neurol Neurosurg Psychiatry 1975;38:1033-35
18. Nappi G, Alfonsi E, Bono G, et al. Goldenhar-Gorlin syndrome: a case with congenital trigeminal neuropathy. Acta Neurol (Napoli) 1983;5:368-72

19. Villanueva O, Atkinson DS, Lambert SR. Trigeminal nerve hypoplasia and aplasia in children with Goldenhar syndrome and corneal hypoesthesia. J AAPOS 2005;9:202-04

20. Miller MT. Association of Duane retraction syndrome with craniofacial malformations. J Craniofac Genet Dev Biol Suppl 1985;1: $273-82$

21. Dastur YK, Trivedi H, Tapaswi N, et al. Goldenhar's syndrome with unilateral Duane retraction syndrome and "butterfly" vertebra. Indian J Ophthalmol 1985;33:187-89

22. Mansour AM, Wang F, Henkind $P$, et al. Ocular findings in the facioauriculovertebral sequence (Goldenhar-Gorlin syndrome). Am J Ophthalmol 1985;100:555-59

23. Miller NR, Kiel SM, Green WR, et al. Unilateral Duane retraction syndrome (type 1). Arch Ophthalmol 1982;100:1468

24. Baum J, Feingold M. Ocular aspects of Goldenhar syndrome. Am J Ophthalmol 1973;75:250-57

25. Mittelman D, Herbst R, Miller M. The many faces of Goldenhar syndrome. In: Proceedings of the Annual Meeting of the American Medical Association, New York. June 1973

26. Vélez G. Duane's retraction syndrome associated with Goldenhar's syndrome. Am J Ophthalmol 1970;70:945-46

27. Yan F, Li J, Xian J, et al. The cochlear nerve canal and internal auditory canal in children with normal cochlea but cochlear nerve deficiency. Acta Radiol 2013;54:292-98

28. Panzer S, Cohen M, Esch U, et al. Radiological evidence of Goldenhar syndrome in a paleopathological case from a South German ossuary. Homo 2008;59:453-61

29. Verzijl HT, Valk J, de Vries R, et al. Radiologic evidence for absence of the facial nerve in Möbius syndrome. Neurology 2005;64: 849-55

30. Joshi VM, Navlekar SK, Kishore GR, et al. CT and MR imaging of the inner ear and brain in children with congenital sensorineural hearing loss. Radiographics 2012;32:683-98

31. Vincenti V, Ormitti F, Ventura E, et al. Cochlear implantation in children with cochlear nerve deficiency. Int J Pediatr Otorhinolaryngol 2014;78:912-17

32. Warren FM 3rd, Wiggins RH 3rd, Pitt C, et al. Apparent cochlear nerve aplasia: to implant or not to implant? Otol Neurotol 2010;31:1088-94

33. Deggouj N, Gersdorff M, Garin P, et al. Today's indications for cochlear implantation. B-ENT 2007;3:9-14 